\title{
Patterns of Synchrony in Lattice Dynamical Systems
}

\author{
Fernando Antoneli* \\ Ana Paula S. Dias ${ }^{\dagger}$ \\ Martin Golubitsky \\ Yunjiao Wang ${ }^{\S}$
}

December 14, 2004

\begin{abstract}
From the point of view of coupled systems developed by Stewart, Golubitsky, and Pivato, lattice differential equations consist of choosing a phase space $\mathbf{R}^{k}$ for each point in a lattice and a system of differential equations on each of these spaces $\mathbf{R}^{k}$ such that the whole system is translation invariant. The architecture of a lattice differential equation is the specification of which sites are coupled to which (nearest neighbor coupling is a standard example). A polydiagonal is a finite-dimensional subspace of phase space obtained by setting coordinates in different phase spaces equal; a pattern of synchrony is a polydiagonal that is flow-invariant for every lattice differential equation with a given architecture. We prove that every pattern of synchrony for a fixed architecture in planar lattice differential equations is spatially doubly periodic assuming that the couplings are sufficiently extensive. For example, nearest and next nearest neighbor couplings are needed for square and hexagonal couplings, and a third level of coupling is needed for the corresponding result to hold in rhombic and primitive cubic lattices. On planar lattices this result is known to fail if the network architecture consists only of nearest neighbor coupling. The techniques we develop to prove spatial periodicity and finiteness can be applied to other lattices.
\end{abstract}

\footnotetext{
*Department of Applied Mathematics, University of São Paulo, São Paulo, SP 05508-090, Brazil.

${ }^{\dagger}$ Centro de Matemática, Universidade do Porto, Porto, 4169-007, Portugal

¥Department of Mathematics, University of Houston, Houston, TX 77204-3008, USA.

${ }^{\S}$ Department of Mathematics, University of Houston, Houston, TX 77204-3008, USA.
} 


\section{Introduction}

Many physical and biological systems can be modelled by networks of systems of differential equations. Networks of differential equations possess additional structure, namely, canonical observables - the dynamical behavior of the individual network nodes [4]. These observables can be compared, revealing such features as synchrony or in periodic solutions specified phase-relations. These features are important in many applications and any theoretical treatment of network dynamics must take this additional structure into account.

Stewart, Golubitsky, Pivato, and Török [5,6] formalize the concept of a coupled cell network, where a cell is a system of ordinary differential equations (ODEs) and a coupled cell system consists of cells whose equations are coupled. Stewart et al. define the architecture of coupled cell networks and develop a theory that shows how network architecture leads to synchrony. The architecture of a coupled cell network is a graph that indicates which cells have the same phase space, which cells are coupled to which, and which couplings are the same. See also the development by Field [2].

In this paper we study properties of synchrony in lattice differential equations. We use a strong form of network synchrony, namely, robust synchrony, which we now define. A polydiagonal $\Delta$ is a subspace of the phase space of a coupled cell system that is defined by equality of cells coordinates. The polydiagonal $\Delta$ is robustly polysynchronous if $\Delta$ is flowinvariant for every coupled cell system with the given network architecture. Solutions in a flow-invariant $\Delta$ have a collection of coordinates equal for all time. If we color two cells the same when the coordinates are equal, then we can associate robustly polysynchronous polydiagonals with patterns of synchrony.

Stewart et al. [6, Theorem 6.1] prove that a polydiagonal is robustly polysynchronous if and only if the coloring given by coloring cells that have the same coordinates with the same color is balanced. (The definition of balanced is given in Definition 2.9.) Thus, classifying robustly polysynchronous polydiagonals is equivalent to the combinatorial question of classifying balanced colorings.

A lattice dynamical system is a coupled cell system with cells indexed by a lattice $\mathcal{L}$. Each cell has a finite set of cells $I(c)$ that are coupled to $c$. A standard example of network architecture is given by nearest neighbor coupling in which case $I(c)$ consists of those cells in the lattice that are nearest to $c$. A lattice differential equation has the form

$$
\dot{x}_{c}=g\left(x_{c}, x_{I(c)}\right) \quad c \in \mathcal{L}
$$


where $x_{c} \in \mathbf{R}^{n}, I(c)=\left\{c_{1}, \ldots, c_{k}\right\}, x_{I(c)}=\left(x_{c_{1}}, \ldots, x_{c_{k}}\right) \in\left(\mathbf{R}^{n}\right)^{k}$ and $g:\left(\mathbf{R}^{n}\right)^{k+1} \rightarrow \mathbf{R}^{n}$.

Golubitsky, Nicol, and Stewart [3] give an infinite class of two-color patterns of synchrony on square lattice systems with nearest neighbor coupling. Wang and Golubitsky [7] classify all possible two-color patterns of synchrony of square and hexagonal lattice differential equations with two different architectures - nearest neighbor coupling (NN) and both nearest neighbor and next nearest neighbor coupling (NNN). It follows from these results that with NNN architecture balanced two-colorings are finite in number and spatially doubly-periodic. Thus, there is a profound difference between balanced two-colorings in the NN and NNN cases: one classification is finite, the other is infinite; one set has spatially periodic and nonperiodic colorings, the other has only periodic colorings.

In this paper we show that each balanced $k$-coloring on a square and hexagonal lattice with NNN architecture is spatially periodic and that there are only a finite number of $k$ colorings for each $k$. See Theorem 4.1. The techniques we develop are general enough to prove similar theorems for other lattices; the general principle seems to be that if there is enough coupling, then balanced $k$-colorings are spatially periodic.

In Section 2 we discuss the general structure of lattice differential equations. The techniques that we use to prove spatial periodicity and finiteness (namely, the notions of 'window' and 'determining boundaries') are discussed in Section 3. The theorems on planar lattices are given in Section 4 and a cubic lattice is discussed in Section 5.

\section{Lattice Dynamical Systems}

In this section we define what we mean by a lattice dynamical system. We begin by defining a coupled cell system abstractly as in [5].

Definition 2.1 A coupled cell network $G$ consists of:

(a) A countable set $\mathcal{C}$ of cells.

(b) An equivalence relation $\sim_{C}$ on cells in $\mathcal{C}$.

(c) A countable set $\mathcal{E}$ of edges or arrows.

(d) An equivalence relation $\sim_{E}$ on edges in $\mathcal{E}$. 
(e) (Local finiteness) There is a head map $\mathcal{H}: \mathcal{E} \rightarrow \mathcal{C}$ and a tail map $\mathcal{T}: \mathcal{E} \rightarrow \mathcal{C}$ such that for every $c \in \mathcal{C}$ the sets $\mathcal{H}^{-1}(c)$ and $\mathcal{T}^{-1}(c)$ are finite.

We also require a consistency condition:

(f) Equivalent arrows have equivalent tails and heads; that is, if $e_{1} \sim_{E} e_{2}$ in $\mathcal{E}$, then $\mathcal{H}\left(e_{1}\right) \sim_{C} \mathcal{H}\left(e_{2}\right)$ and $\mathcal{T}\left(e_{1}\right) \sim_{C} \mathcal{T}\left(e_{2}\right)$.

Remark 2.2 Associated with each cell $c \in \mathcal{C}$ is a set of edges that represent couplings into c. In the abstract setting of [5] multiple connections between cells and self-coupling are permitted. Because of this it is most natural to think of inputs as arrows. This generality is not needed in our discussion of lattice dynamical systems; so we can identify input arrows with their tail cells, as was done originally in [6].

Definition 2.3 Let $c \in \mathcal{C}$. The input set of $c$ is

$$
I(c)=\mathcal{T}\left(\mathcal{H}^{-1}(c)\right)
$$

An element of the finite set $I(c)$ is called an input cell of $c$.

Two input sets are isomorphic if there is a bijection between the input sets that preserves coupling types. A coupled cell network is homogeneous if the input sets of all cells are isomorphic.

An $n$-dimensional lattice $\mathcal{L}$ is a subset of $\mathbf{R}^{n}$ of the form

$$
\mathcal{L}=\left\{\alpha_{1} v_{1}+\cdots+\alpha_{n} v_{n}: \alpha_{i} \in \mathbf{Z}\right\}
$$

where $\left\{v_{1}, \ldots, v_{n}\right\}$ is a set of linearly independent vectors in $\mathbf{R}^{n}$ called the generators of the lattice $\mathcal{L}$. Note that $\mathcal{L}$ is a discrete subgroup of $\mathbf{R}^{n}$.

Definition 2.4 We call a lattice $\mathcal{L}$ Euclidean if it satisfies

(a) All generators of $\mathcal{L}$ have the same length.

(b) The generators of $\mathcal{L}$ are exactly those lattice vectors that are nearest to the origin in Euclidean distance. 
Euclidean lattices are the most relevant for applications of bifurcation theory [4]. Planar square and hexagonal lattices are Euclidean. The generators $v_{1}, v_{2}$ of a rhombic lattice can be assumed to be in the first quadrant. A rhombic lattice satisfies (b) only when the angle between $v_{1}$ and $v_{2}$ is greater than $\pi / 3$.

Let $r_{0}<r_{1}<\cdots$ be the possible lengths of vectors in a fixed lattice $\mathcal{L}$. We can partition the vectors in $\mathcal{L}$ by length as follows. Let

$$
J_{i}=\left\{v \in \mathcal{L}:|v|=r_{i}\right\}
$$

The vectors in $\mathcal{L}$ can be divided into classes of neighbors as follows. The nearest neighbors to a vector $c \in \mathcal{L}$ is the set of vectors $\left\{c+v: v \in J_{1}\right\}$. The next nearest neighbors to $c$ is the set of vectors $\left\{c+v: v \in J_{2}\right\}$. The $p^{\text {th }}$ nearest neighbors to $c$ is the set of vectors $\left\{c+v: v \in J_{p}\right\}$.

Definition 2.5 An n-dimensional lattice network consists of:

(a) An $n$-dimensional lattice $\mathcal{L}$.

(b) A homogeneous coupled cell system $G_{\mathcal{L}}$ whose cells are indexed by $\mathcal{L}$.

(c) The set $I(0)=J_{1} \cup \cdots \cup J_{p}$ for some $p$.

(d) The edge type of two cells in the same class of neighbors is the same and each class of neighbors corresponds to a different edge type.

We say that a lattice in which the cells are coupled to neighbors of order $p$ is a lattice with $p$-th nearest neighbor coupling. In particular, if $p=1$ we have a lattice with nearest neighbor coupling and if $p=2$ we have a lattice with nearest and next nearest neighbor coupling. Figure 1 shows examples of two-dimensional lattice networks.

Remarks 2.6 (a) Lattice networks are bidirectional, that is, for each arrow from $c$ to $d$ there is an arrow of the same type from $d$ to $c$. This follows from Definition 2.5.

(b) The symmetry group of the lattice is the symmetry group of the lattice network. In particular, translations by any vector in the lattice is a symmetry of the lattice network.

Example 2.7 Up to equivalence there is exactly one lattice $\mathcal{L}$ in $\mathbf{R}$. If we normalize the length of the generator of the lattice to be $1, \mathcal{L} \cong \mathbf{Z}$. In a network defined on $\mathbf{Z}$ each cell $i$ has exactly two neighbors of order $p$, namely the left $(i-p)$ and the right $(i+p)$. 

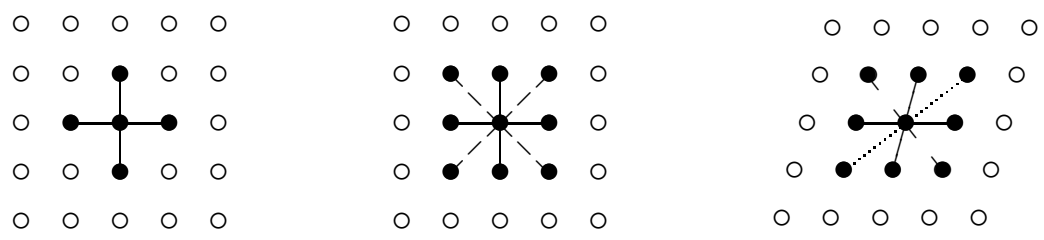

Figure 1: (Left) square lattice network with nearest neighbor coupling (solid lines). (Center) square lattice network with nearest neighbor and next nearest neighbor coupling (dashed lines). (Right) rhombic lattice network with nearest neighbor, next nearest neighbor, and next next nearest neighbor coupling (dotted lines).

Definition 2.8 An $n$-dimensional lattice dynamical system is a system of ordinary differential equations associated to a $n$-dimensional lattice network $G_{\mathcal{L}}$ given by

$$
\dot{x_{c}}=f\left(x_{c}, x_{I(c)}\right) \quad c \in \mathcal{L}
$$

where $x_{c} \in \mathbf{R}^{k}, I(c)=\left\{c_{1}, \ldots, c_{\ell}\right\}, x_{I(c)}=\left(x_{c_{1}}, \ldots, x_{c_{\ell}}\right) \in \mathbf{R}^{k \ell}$ and the map $f: \mathbf{R}^{k(\ell+1)} \rightarrow$ $\mathbf{R}^{k}$ is smooth. The corresponding vector field is said to be $G_{\mathcal{L}^{-}}$admissible.

A pattern of synchrony in a lattice dynamical system is identified with a robustly polysynchronous equivalence relation, that is, an equivalence relation $\bowtie$ on the cells such that the associated polydiagonal

$$
\Delta_{\bowtie}=\left\{x \in \mathbf{R}^{k(\ell+1)}: x_{c}=x_{d} \text { whenever } c, d \in \mathcal{L} \text { and } c \bowtie d\right\}
$$

is flow-invariant under every $G_{\mathcal{L}^{-}}$admissible vector field. It has been proved $[5,6]$ that an equivalence relation is robustly polysynchronous if and only if it is balanced.

Suppose that we have a finite number $\ell$ of $\bowtie$-equivalence classes and we color the cells in the lattice so that two cells have the same color precisely when they are in the same $\bowtie$-equivalence class, that is, an equivalence relation can be represented by an $\ell$-coloring of the cells. Now let $K_{1}, \ldots, K_{\ell}$ be the colors of an $\ell$-coloring of a lattice network $G_{\mathcal{L}}$.

Definition 2.9 The $\ell$-coloring is balanced if and only if each cell of color $K_{i}$ receives the same number of inputs from cells of color $K_{j}(j=1, \ldots, \ell)$ of each edge type. 


\section{Techniques for Proving Spatial Periodicity}

Definition 3.1 Let $G_{\mathcal{L}}$ be a lattice network and let $U \subset \mathcal{L}$ be a subset. The closure of $U$ consists of all cells that are connected by some arrow to a cell in $U$, that is,

$$
\operatorname{cl}(U)=\{\mathcal{T}(e): e \in \mathcal{E} \text { and } \mathcal{H}(e) \in U\} .
$$

The boundary of $U$ is the set

$$
\operatorname{bd}(U)=\operatorname{cl}(U) \backslash U
$$

For each Euclidean lattice network $G_{\mathcal{L}}$ there is a natural expanding sequence of finite subsets that covers the lattice. Let

$$
W_{0}=\{0\} \quad \text { and } \quad W_{i+1}=\operatorname{cl}\left(W_{i}\right)
$$

for $i \geqslant 0$. Since the input set of each cell contains the generators of the lattice, we have

$$
\mathcal{L}=W_{0} \cup W_{1} \cup \cdots
$$

It follows that for any coloring of a lattice $\mathcal{L}$ by $k$ colors, there is some $j$ such that all $k$ colors are represented by cells in $W_{j}$. In fact, more is true for balanced colorings.

Lemma 3.2 Let $G_{\mathcal{L}}$ be a lattice network with a balanced $k$-coloring. Then $W_{k-1}$ contains all $k$ colors.

Proof: We claim that if $\ell<k$, then $W_{\ell}$ contains at least $\ell+1$ colors. The proof proceeds by induction on $W_{\ell} . W_{0}=\{0\}$ contains one cell and one color.

Assume that the statement is true for $\ell<k-1$; we prove that it is also true for $\ell+1$. Suppose that the number $m$ of colors contained in $W_{\ell+1}=\operatorname{cl}\left(W_{\ell}\right)$ is the same as the number of colors in $W_{\ell}$. Then every cell $c \in W_{\ell+1}$ has a color that is the same as the color of a cell $d$ in $W_{\ell}$. So, all cells connected to $d$ lie in $W_{\ell+1}$ and are colored by the $m$ colors. Therefore, balanced implies that the cells connected to $c$ must also be colored by one of the $m$ colors. It follows that the cells in $W_{\ell+2}=\mathrm{cl}\left(W_{\ell+1}\right)$ are also colored by these $m$ colors. By induction the entire lattice is colored by $m$ colors; hence $m=k$. So if $m<k$, the number of colors in $W_{\ell+1}$ must be greater than the number of colors in $W_{\ell}$. That is, $W_{\ell+1}$ contains at least $\ell+2$ colors. It follows that $W_{k-1}$ contains all $k$ colors. 
Definition 3.3 Let $G_{\mathcal{L}}$ be a lattice network and let $U \subset \mathcal{L}$ be a subset of cells. We say that $U$ is connected if for every pair of cells $c, d \in U$ there is a sequence of cells $c=e_{1}, e_{2}, \ldots, e_{j}=$ $d \in U$ such that $e_{i} \in I\left(e_{i+1}\right)$ for all $i=1, \ldots, j-1$.

Definition 3.4 Let $G_{\mathcal{L}}$ be a lattice network and let $U \subset G_{\mathcal{L}}$ be a finite connected set.

(a) A cell $c \in \operatorname{bd}(U)$ is 1-determined if there is a cell $d \in U$ such that $c$ is in the input set of $d$ and each cell in the input set of $d$ that has the same coupling type as $c$, except $c$ itself, belongs to $U$.

(b) A cell $c \in \operatorname{bd}(U)$ is $p$-determined, where $p>1$ if there is a cell $d \in U$ such that $c$ is in the input set of $d$ and each cell in the input set of $d$ that has the same coupling type as $c$, except $c$ itself, either belongs to $U$ or belongs to $\mathrm{bd}(U)$ and is $q$-determined for some $q<p$.

(c) A cell $c \in \operatorname{bd}(U)$ is determined if it is $p$-determined for some $p$.

(d) The set $U$ determines its boundary if all cells in $\operatorname{bd}(U)$ are determined.

Definition 3.5 Let $G_{\mathcal{L}}$ be a lattice network. Then the set $W_{i_{0}}$ is a window if $W_{i}$ determines its boundary for all $i \geqslant i_{0}$.

Remark 3.6 Note that if there are no 1-determined cells then, by induction, there are no $p$-determined cells for any $p$. In particular, if there are no 1-determined cells, then windows do not exist.

Example 3.7 Let $\mathcal{L}$ be the square lattice of length 1 which we can identify with $\mathbf{Z}^{2}$. Let $G_{\mathcal{L}}$ be the associated lattice network such that each cell has four nearest neighbors at distance 1. See Figure 1 (left). This network has no window, as we show. (Note that in this case, it is shown in [7] that there are infinitely many balanced 2-colorings.)

We claim that no set $W_{i}$ is a window. By Remark 3.6 it is sufficient to show that there are no 1-determined cells. For example, consider $W_{2}$ and its boundary (Figure 2). Since the cells on the boundary are in a diagonal line it is not possible for them to be the only cell in the input set of a cell in $W_{2}$ that is not in $W_{2}$. Note that when $i>2$ the set $W_{i}$ has the same "diamond shape" as $W_{2}$. So there are no 1-determined cells in bd( $\left.W_{i}\right)$. By Remark 3.6, this network has no window. 


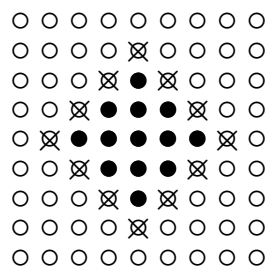

Figure 2: The set $W_{2}$ (black cells) and its boundary (white cells with a cross).

Example 3.8 Let $G_{\mathcal{L}}$ be the lattice network associated to the square lattice $\mathcal{L}=\mathbf{Z}^{2}$ such that each cell has four nearest neighbors at distance 1 and four next nearest neighbors at distance $\sqrt{2}$. See Figure 1 (center).

Let $W_{0}, W_{1}, \ldots$ the sequence of sets generated by cell 0 . It is clear that each set $W_{i}$ is a square of size $2 i+1$. The size of a square is the number of cells in one (and hence all) of its sides.

We show that the sets $W_{i}$ for $i \geqslant 2$ determine their boundaries. To show this we just need (by symmetry) to analyze one of the corners of such a square because all the cells on each side, except the last two on both extremes, are 1-determined since they are the only nearest neighbor outside the square (Figure 3).

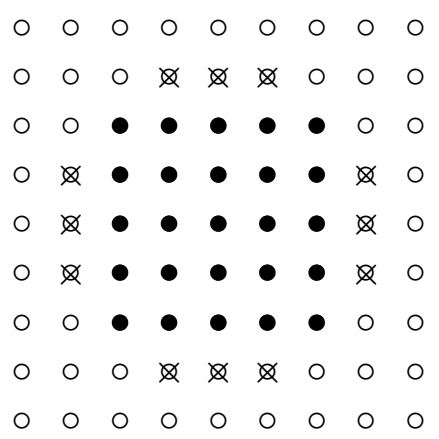

Figure 3: The set $W_{2}$ (black cells) and the 1-determined cells of its boundary.

The three cells in the corners of the square are 2-determined using the next nearest neighbors coupling as long as the square has size greater than 3. See Figure 4.

Definition 3.9 Let $G_{\mathcal{L}}$ be a lattice network and let $U \subset \mathcal{L}$ be a subset. The interior of $U$ consists of all cells $c \in U$ such that any cell connected to $c$ is also in $U$, that is,

$$
\operatorname{int}(U)=\{c \in U: I(c) \subset U\}
$$




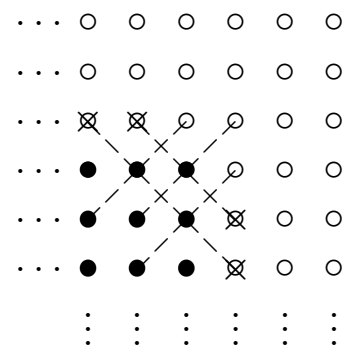

Figure 4: The corner of a set $W_{i}$ (black cells), the 1-determined cells (white cells with a cross) and 2-determined cells (white cells connected to 1-determined cells by dashed lines).

Lemma 3.10 Let $G_{\mathcal{L}}$ be a lattice network where $\mathcal{L}$ is an Euclidean lattice and assume that $W_{i_{0}}$ is a window. Suppose that a balanced $k$-coloring restricted to $\operatorname{int}\left(W_{i}\right)$ for some $i \geqslant i_{0}$ contains all $k$ colors. Then the $k$-coloring is uniquely determined on the whole lattice by its restriction to $W_{i}$.

Proof: Let $K$ be a balanced $k$-coloring. Then for any two cells $c$ and $d$ of the same color, there is a bijection $\beta: I(c) \rightarrow I(d)$ that preserves arrow type and color. So if we know the colors of all cells in $I(c)$ and we know the colors of all cells except one in $I(d)$, then the fact that the coloring is balanced tells us what the color of the last cell in $I(d)$ must be.

Suppose that $c \in \operatorname{bd}\left(W_{i}\right)$ and that $c$ is 1-determined. Then there exists $d \in W_{i}$ and $c \in I(d)$ such that all other input cells in $I(d)$ that have the same coupling type as $c$ are in $W_{i}$. Since $\operatorname{int}\left(W_{i}\right)$ contains all $k$ colors, there exists a cell $e \in \operatorname{int}\left(W_{i}\right)$ with the same color as $d$. Since all neighbors of $e$ are in $W_{i}$ (by definition of interior), their colors are known. In particular, the colors of the cells in $I(d)$ that have the same coupling type as $c$ are known except for the color of $c$. Now we apply the reasoning in the previous paragraph to deduce the color of cell $c$.

Assume that the colors of all $q$-determined cells in $\operatorname{bd}\left(W_{i}\right)$ when $q<p$ have been determined. Suppose $c \in \operatorname{bd}\left(W_{i}\right)$ is $p$-determined. Then there exists $d \in W_{i}$ and $c \in I(d)$ such that all other input cells in $I(d)$ that have the same coupling type as $c$ are in $W_{i}$ or are $q$-determined for some $q<p$. We use the same argument as in the previous paragraph to deduce the color of cell $c$. Since $\mathrm{bd}\left(W_{i}\right)$ is finite and $W_{i}$ determines its boundary, this process ends when all the cells in $\mathrm{bd}\left(W_{i}\right)$ are colored. Hence, the balanced coloring has been extended from $W_{i}$ to $W_{i+1}$. 
Finally, we continue inductively to color $W_{i+\ell}$ for $\ell \geqslant 1$. It follows that the balanced $k$-coloring restricted to $W_{i}$ can be uniquely extended to the whole lattice.

Theorem 3.11 Let $\mathcal{L}$ be an Euclidean lattice and $G_{\mathcal{L}}$ a lattice network with a window. Fix $k \geqslant 1$. Then there are a finite number of balanced $k$-colorings on $\mathcal{L}$ and each balanced $k$-coloring is spatially multiply-periodic.

Proof: Let $W_{j}$ be a window for $G_{\mathcal{L}}$ where $j \geqslant k$. Since there is only a finite number of possible ways to distribute $k$ colors on the cells in $W_{j}$ it follows that there are only a finite number of balanced $k$-colorings restricted to $W_{j}$. Moreover, by Lemma 3.2, the interior of $W_{j}$ contains all $k$-colors. Lemma 3.10 states that any balanced $k$-coloring restricted to $W_{j}$ extends uniquely to the whole lattice. Therefore, the number of balanced $k$-colorings of $G_{\mathcal{L}}$ is finite.

Let $K$ be a balanced $k$-coloring on $G_{\mathcal{L}}$ and let $v \in \mathcal{L}$. Let $T_{v}(K)$ be the coloring obtained by shifting the coloring $K$ by $v$, that is, the color of cell $c$ in $T_{v}(K)$ is the same as the color of cell $c-v$ in $K$. Since translations are symmetries of the lattice network $T_{v}(K)$ is also a balanced coloring. It also follows by symmetry that the subset $T_{v}\left(W_{j}\right)=\left\{c+v: c \in W_{j}\right\}$ has the window property that balanced $k$-colorings restricted to $T_{v}\left(W_{j}\right)$ uniquely extend to the whole plane.

Let $v$ be a generator of the lattice and consider all translates of $W_{j}$ in the direction of $v$. Since there are only a finite number of balanced $k$-colorings and an infinite number of translates of $W_{j}$, there must be at least two translates $W_{j}^{1}$ and $W_{j}^{2}$ exhibiting exactly the same balanced $k$-coloring. Therefore the balanced $k$-colorings determined by $W_{j}^{1}$ and $W_{j}^{2}$ are the same. Since that translation of a balanced $k$-coloring is again a balanced $k$-coloring, it follows that the translation that takes $W_{k}^{1}$ to $W_{k}^{2}$ leaves the balanced $k$-coloring invariant and hence it is periodic in the direction of $v$. The same argument can be applied to all the generators of the lattice, thus all balanced $k$-colorings are spatially multiply-periodic.

The fundamental property that we have identified in the course of the proof of the theorems in this section is determinacy, which is related to the architecture of the network defined by the choice of the structure of the input set.

Example 3.12 Consider the one-dimensional lattice $\mathcal{L}=\mathrm{Z}$. Let $G_{\mathcal{L}}$ be the lattice network with nearest neighbor coupling. The input set of a cell $c$ consists of $c$ plus its left and right neighbors. Let $W_{0}, W_{1}, \ldots$ be the sequence of sets defined in (3.1). Then 
$W_{i}=\{-i, \ldots, 0, \ldots, i\}$ is an interval of $2 i+1$ consecutive cells. Note that the boundary of any interval has two cells that are not in the interval and both of them are 1-determined. Therefore, the sets $W_{i}$ for $i \geq 1$ are windows. Theorem 3.11 implies the finiteness of balanced $k$-colorings and spatial periodicity of all balanced $k$-colorings for the one-dimensional lattice network with nearest neighbor coupling. This special case is proved directly in [1].

\section{Planar Lattices}

Our main result about balanced colorings of planar lattice networks is the following.

Theorem 4.1 Let

$$
\mathcal{L}=\{\alpha u+\beta v: \alpha, \beta \in \mathbf{Z}\}
$$

be a planar lattice, where the generators $u$ and $u$ are norm 1 linearly independent vectors. Assume that the angle $\theta$ between $u$ and $v$ satisfies

$$
\frac{\pi}{3} \leq \theta \leq \frac{\pi}{2}
$$

Let $G_{\mathcal{L}}$ be the associated network such that the input set of each cell c contains cells whose distance from $c$ is less than or equal to $|u+v|$. Then for each $k>0$ the network $G_{\mathcal{L}}$ admits only a finite number of balanced $k$-colorings each of which is spatially doubly-periodic.

Remark 4.2 Theorem 4.1 covers three types of lattice:

(a) square lattice: $u=(1,0)$ and $v=(0,1)$

(b) hexagonal lattice: $u=(1,0)$ and $v=(1, \sqrt{3}) / 2$

(c) rhombic lattice: $u=(1,0)$ and $v=(\cos \theta, \sin \theta)$ where $\frac{\pi}{3}<\theta<\frac{\pi}{2}$.

For each of these lattices we define the critical distance as $|u+v|$. The couplings allowed by the critical distance are nearest and next nearest neighbor for all three lattices and next next nearest neighbor for the rhombic lattices.

Proof: It is sufficient to show that the three types of lattices mentioned in the Remark 4.2 have windows. More precisely, let $W_{0}, W_{1}, \ldots$ be the sets defined in (3.1) for one of the 
lattices satisfying the hypothesis of the theorem. We shall prove that $W_{i}$ determines its boundary for all $i \geqslant 2$ and is a window. The conclusion follows from Theorem 3.11.

First, let $\mathcal{L}$ be the square lattice. We already have shown in Example 3.8 that for all $i \geqslant 2$ the set $W_{i}$ determines its boundary.

Second, let $\mathcal{L}$ be a rhombic lattice with $\frac{\pi}{3}<\theta<\frac{\pi}{2}$. Since this lattice is a deformation of the square lattice, the same argument that is used in Example 3.8 shows that $W_{i}$ determines its boundary for all $i \geqslant 2$. The only new issue is that the set of next nearest neighbors has four elements in the square lattice breaks into two sets of two elements each in the rhombic lattice. See Figure 1 (right).

Third, let $\mathcal{L}$ be the hexagonal lattice. The input set of a cell $c$ in the hexagonal lattice with nearest and next nearest neighbor coupling has 12 cells: 6 nearest neighbors at distance 1 from $c$ and 6 next nearest neighbors at distance $\sqrt{3}$ from $c$ (Figure 5 ).

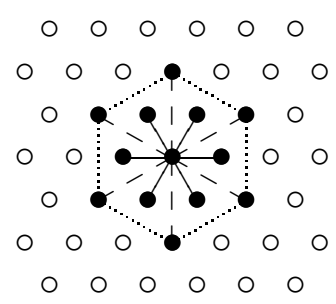

Figure 5: Hexagonal lattice network. Nearest neighbor (solid lines) and next nearest neighbor (dashed lines) coupling. The dotted lines show the hexagonal region $W_{1}$.

The set $W_{i+1} \backslash W_{i}$ is a hexagonal annulus surrounding $W_{i}$. Indeed, the cells in the input set of one cell $c$ in $W_{i}$ are within a distance less or equal than $\sqrt{3}$ from $c$, so they must lie inside this region. See Figure 6. Another observation is that the three lines through 0 and the next nearest neighbors of 0 divide each set $W_{i}$ into six sectors. Since rotations by $\pi / 3$ are symmetries of the lattice, we can restrict the analysis to one of these sectors.

In the hexagonal lattice the boundaries of the sets $W_{i}$ in a given sector consists of three lines of cells. See Figure 7. Note that cells on the first line of $W_{i}$ are nearest neighbors of cells on the second line of $W_{i-1}$; cells on the second line of $W_{i}$ are nearest neighbors of the cells on the third line of $W_{i-1}$; and cells on the third line of $W_{i}$ are nearest neighbors of the cells on the first line of $W_{i}$.

The first line of the boundary of a set $W_{i}$ is 1-determined. This follows from the fact that a cell $c$ in the first line of $\operatorname{bd}\left(W_{i}\right)$ is a nearest neighbor of a cell $d$ in the second line of $W_{i}$ and all other nearest neighbors of $d$ are in $W_{i}$. See Figure 8. The same argument shows 


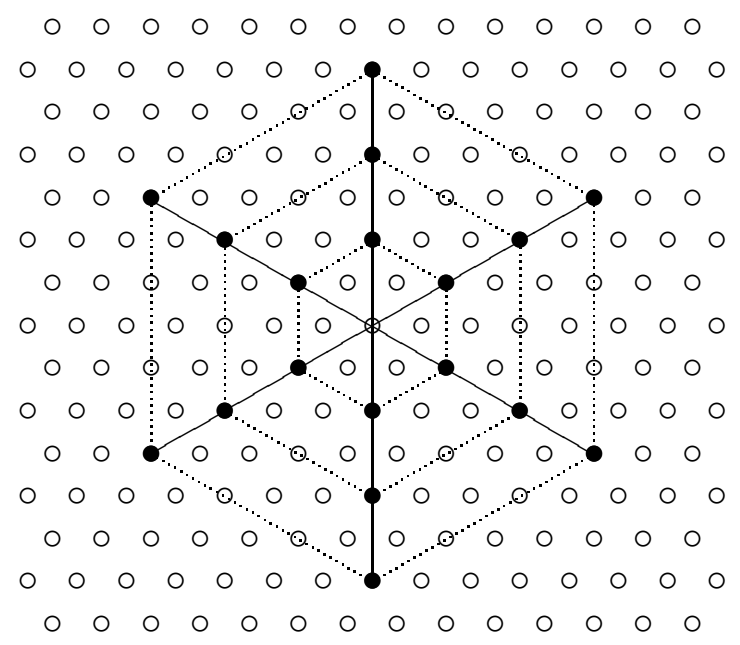

Figure 6: The next nearest neighbors of 0 and the sets $W_{1}, W_{2}$ and $W_{3}$ (hexagonal regions defined by dotted lines). The six sectors defined by the next nearest neighbors are separated by solid lines.

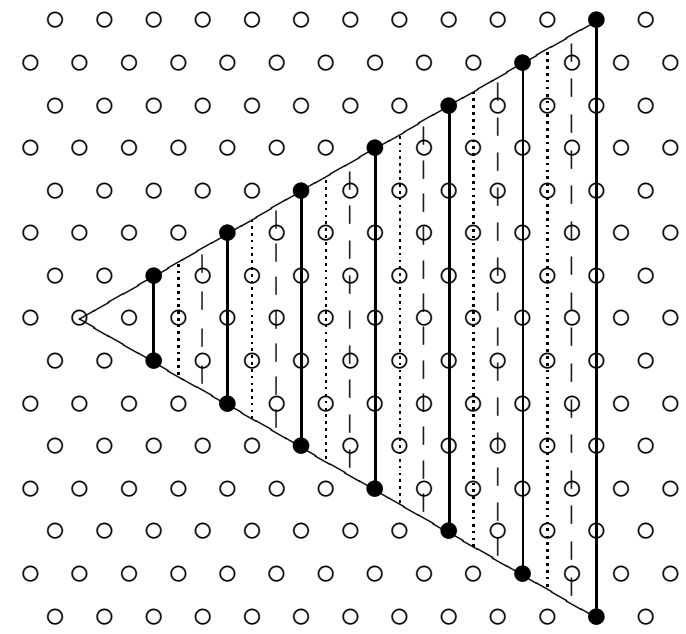

Figure 7: One sector of the sets $W_{i+1} \backslash W_{i}$ with the three lines of cells connected by dots, dashes, and solid.

that cells in the second line, with the exception of the two cells nearest the sector boundary, are 2-determined; and cells in the third line of one sector, with the exception of the two cells on the sector boundary and the two cells nearest the sector boundary, are 3-determined. So far, we have shown that, except for six cells near or on the boundary of the sector, all cells are determined. 


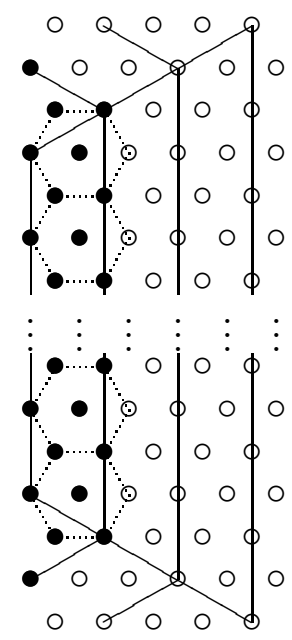

Figure 8: One sector of the set $W_{i}$ (black cells) and a sector of its boundary $\operatorname{bd}\left(W_{i}\right)$. The first line of $\mathrm{bd}\left(W_{i}\right)$ is 1-determined.

Reflections allow us to restrict ourselves to cells near one of corner of a sector. Thus we must show that the three remaining cells are determined. We now assume that $i \geqslant 2$.

To see that cell $c_{1}$ near the sector boundary on the second line of $\operatorname{bd}\left(W_{i}\right)$ is determined, consider the next nearest neighbors of the cell $d_{1}$ near the sector boundary on the second line of $\operatorname{bd}\left(W_{i-1}\right)$. Since $c_{1}$ is the only next nearest neighbor of $d_{1}$ that has not yet been determined, $c_{1}$ is determined. See Figure $9(\mathrm{a})$.

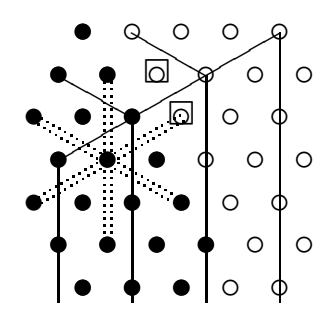

(a)

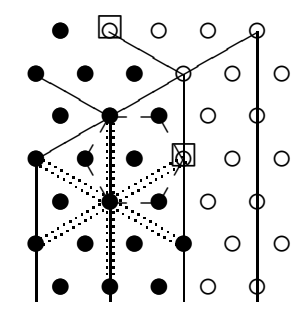

(b)

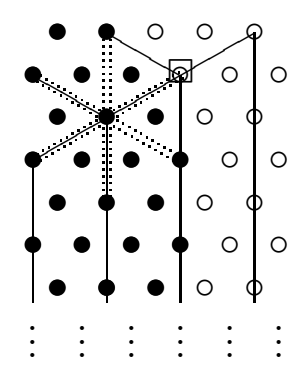

(c)

Figure 9: The corner of one sector of the set $W_{i}$. The three remaining cells are determined.

To see that cell $c_{2}$ on the third line but not on the sector boundary is determined, consider the nearest neighbors of cell $d_{2}$ nearest the second line of $\operatorname{bd}\left(W_{i}\right)$. Since $c_{2}$ is the only nearest neighbor of $d_{2}$ that has not yet been determined, $c_{2}$ is determined. See Figure $9(\mathrm{~b})$. 
To see that cell $c_{3}$ on the third line of $\mathrm{bd}\left(W_{i}\right)$ and the sector boundary is determined, consider the next nearest neighbors of cell $d_{3}$ on the sector boundary and the third line of $\operatorname{bd}\left(W_{i-1}\right)$. Since $c_{3}$ is the only next nearest neighbor of $d_{3}$ that has not yet been determined, $c_{3}$ is also determined. See Figure $9(\mathrm{c})$.

\section{The Cubic Lattice}

In this section we show that our techniques can also work on three-dimensional lattices by considering the standard (or primitive) cubic lattice $\mathcal{L}=\mathbf{Z}^{3}$. This lattice is the direct generalization to $\mathbf{R}^{3}$ of the linear lattice in $\mathbf{R}$ and the square lattice in $\mathbf{R}^{2}$.

Proposition 5.1 Let $G_{\mathcal{L}}$ be the standard cubic lattice network with nearest, second nearest and third nearest neighbor couplings. Then $W_{s}$ determines its boundary for all $s \geqslant 3$ and $G_{\mathcal{L}}$ admits a window.

Proof: Note that if a cell with coordinates $y=\left(y_{1}, y_{2}, y_{3}\right)$ is in the input set of a cell with coordinates $x=\left(x_{1}, x_{2}, x_{3}\right)$, then the coordinates must satisfy

$$
\left|y_{i}-x_{i}\right| \leqslant 1, \text { for } i=1,2,3
$$

Therefore,

$$
W_{s}=\left\{\left(x_{1}, x_{2}, x_{3}\right):-s \leqslant x_{i} \leqslant s, x_{i} \in \mathbf{Z}\right\}
$$

is the cube centered at the origin whose sides have $2 s+1$ cells. We prove that $W_{s}(s \geqslant 3)$ determines its boundary. Observe that

$$
\begin{aligned}
\operatorname{bd}\left(W_{s}\right) & =\operatorname{cl}\left(W_{s}\right) \backslash W_{s} \\
& =W_{s+1} \backslash W_{s} \\
& =\left\{\left(x_{1}, x_{2}, x_{3}\right) \in W_{s+1}: \exists i \in\{1,2,3\} \text { such that }\left|x_{i}\right|=s+1\right\}
\end{aligned}
$$

By symmetry it is sufficient to prove that all the cells in the set

$$
Q=\left\{\left(s+1, x_{2}, x_{3}\right): 0 \leqslant x_{3} \leqslant x_{2} \leqslant s+1\right\} .
$$

are determined by $W_{s}$. We partition $Q$ into

$$
Q=\left(P_{11} \cup P_{12} \cup P_{13} \cup P_{14}\right) \cup\left(P_{21} \cup P_{22}\right) \cup P_{3}
$$


where

$$
\begin{aligned}
P_{11} & =\left\{\left(s+1, x_{2}, x_{3}\right): 0 \leqslant x_{3} \leqslant x_{2} \leqslant s-1\right\} \\
P_{12} & =\left\{\left(s+1, s, x_{3}\right): 0 \leqslant x_{3} \leqslant s-2\right\} \\
P_{13} & =\{(s+1, s, s)\} \\
P_{14} & =\{(s+1, s, s-1)\} \\
P_{21} & =\left\{\left(s+1, s+1, x_{3}\right): 0 \leqslant x_{3} \leqslant s-1\right\} \\
P_{22} & =\{(s+1, s+1, s)\} \\
P_{3} & =\{(s+1, s+1, s+1)\} .
\end{aligned}
$$

We show that all cells in each of these sets are determined.

$P_{11}$ is 1-determined: Note that cells $\left(s, x_{2}, x_{3}\right)$ with $0 \leqslant x_{3} \leqslant x_{2} \leqslant s-1$ are in $W_{s}$. These cells have six nearest neighbors: $\left(s \pm 1, x_{2}, x_{3}\right),\left(s, x_{2} \pm 1, x_{3}\right)$, and $\left(s, x_{2}, x_{3} \pm 1\right)$. Except for the cell $\left(s+1, x_{2}, x_{3}\right)$, all other nearest neighbors of these cells are in $W_{s}$. Hence, all cells $\left(s+1, x_{2}, x_{3}\right)$ are 1-determined.

$P_{12}$ is 2-determined: Note that cells $\left(s, s-1, x_{3}\right)$ with $0 \leqslant x_{3} \leqslant s-2$ are in $W_{s}$. These cells have 12 next nearest neighbors whose coordinates are:

$$
\begin{aligned}
& \left(s \pm 1,(s-1) \pm 1, x_{3}\right), \quad\left(s \pm 1,(s-1) \mp 1, x_{3}\right), \\
& \left(s \pm 1, s-1, x_{3} \pm 1\right), \quad\left(s \pm 1, s-1, x_{3} \mp 1\right), \\
& \left(s,(s-1) \pm 1, x_{3} \pm 1\right), \quad\left(s,(s-1) \pm 1, x_{3} \mp 1\right) .
\end{aligned}
$$

Except for $\left(s+1, s, x_{3}\right)$, all other next nearest neighbors are in $W_{s} \cup P_{11}$ (or in one of its symmetric images). Thus, all cells $\left(s+1, s, x_{3}\right)$ are 2-determined.

$P_{13}$ is 3-determined: The set $P_{13}$ has one cell $c=(s+1, s, s)$. Note that $d=(s, s-1, s-1)$ is in $W_{s}$ and has $c$ as its next next nearest neighbor. Thus the distance between $c$ and $d$ is $\sqrt{3}$. Since the coordinates of $d$ satisfy (5.1), it follows that, except for $(s+1, s, s)$, all other next next nearest neighbors of $d$ are in $W_{s} \cup P_{11} \cup P_{12}$ (or in one of its symmetric images). Indeed, $(s, s-1, s-1)+(1,1,1)=(s+1, s, s) \notin W_{s} \cup P_{11} \cup P_{12}$ (or one of its symmetric images) and it is a next next nearest neighbor of $d$. Hence $P_{13}$ is 3-determined.

$P_{14}$ is 4-determined: The set $P_{14}$ has one cell $c=(s+1, s, s-1)$. Note that $d=$ $(s, s-1, s-2)$ is in $W_{s}$ and has $c$ as its next next nearest neighbor. This implies that 
the distance between $c$ and $d$ is $\sqrt{3}$. Since the coordinates of $d$ satisfy (5.1), it follows that, except for $(s+1, s, s-1)$, all other next next nearest neighbors of $d$ are in $W_{s} \cup P_{11} \cup P_{12} \cup P_{13}$ (or in one of its symmetric images). Hence, $c$ is 4-determined.

$P_{21}$ is 5-determined: Let $c=\left(s+1, s+1, x_{3}\right)$ where $0 \leqslant x_{3} \leqslant s-1$. Note that $d=\left(s, s, x_{3}\right)$ is in $W$ and has $c$ as its next nearest neighbor. Thus the distance between $c$ and $d$ is $\sqrt{2}$. Since the coordinates of $d$ satisfy (5.1), it follows that, except for $\left(s+1, s+1, x_{3}\right)$, all other next nearest neighbors of $d$ are in $W_{s} \cup P_{11} \cup P_{12} \cup P_{13} \cup P_{14}$ (or in one of its symmetric images). Hence, $P_{21}$ is 5 -determined.

$P_{22}$ is 6-determined: The set $P_{22}$ has one cell $c=(s+1, s+1, s)$. Note that $d=$ $(s, s, s-1)$ is in $W_{s}$ and has $c$ as one of its next next nearest neighbors. Moreover, except for $(s+1, s+1, s)$, all other next next nearest neighbors of $d$ are in $W_{s} \cup P_{11} \cup P_{12} \cup P_{13} \cup P_{14} \cup P_{21}$ (or in one of its symmetric images). Hence, $c$ is 6-determined.

$P_{3}$ is 7-determined: The set $P_{3}$ has one cell $c=(s+1, s+1, s+1)$. Note that $d=(s, s, s)$ is in $W_{s}$ and has $c$ as one of its next next nearest neighbors. Moreover, except for $(s+1, s+$ $1, s+1)$, all other next next nearest neighbors of $d$ are in $W_{s} \cup P_{11} \cup P_{12} \cup P_{13} \cup P_{14} \cup P_{21} \cup P_{22}$ (or in one of its symmetric images). Hence, $P_{3}$ is 7 -determined.

This concludes the proof that $W_{s}$ determines its boundary for all $s \geqslant 3$.

Corollary 5.2 Let $G_{\mathcal{L}}$ be the standard cubic lattice network with nearest, second nearest and third nearest neighbor couplings. Then for each $k>0$ the network $G_{\mathcal{L}}$ admits only a finite number of balanced $k$-colorings each of which is spatially triply-periodic.

\section{Acknowledgments}

FA and APSD thank the Department of Mathematics of the University of Houston for its hospitality. This work was supported in part by NSF Grant DMS-0244529 and ARP Grant 003652-0032-2001. The work of FA was supported in part by a FAPESP Grant 03/12631-3. 


\section{References}

[1] F. Antoneli, A.P.S. Dias, M. Golubitsky, and Y. Wang. Flow invariant subspaces for lattice dynamical systems. In: Workshop on Bifurcation Theory and Spatio-Temporal Pattern Formation in PDE (W. Nagata and N.S. Namachchivaya, eds.) Fields Institute Communications. To appear.

[2] M. Field. Combinatorial dynamics. Dynamical Systems 19 (2004) 217-243.

[3] M. Golubitsky, M. Nicol, and I. Stewart. Some curious phenomena in coupled cell systems. J. Nonlinear Sci. 14 (2) (2004) 119-236.

[4] M. Golubitsky and I. Stewart. The Symmetry Perspective, Progress in Mathematics 200, Birkhäuser, Basel 2002.

[5] M. Golubitsky, I. Stewart, and A. Török. Patterns of synchrony in coupled cell networks with multiple arrows. SIAM J. Appl. Dynam. Sys. 4 (1) (2005). To appear.

[6] I. Stewart, M. Golubitsky, and M. Pivato. Symmetry groupoids and patterns of synchrony in coupled cell networks. SIAM J. Appl. Dynam. Sys. 2 (4) (2003) 609-646.

[7] Y. Wang and M. Golubitsky. Two-color patterns of synchrony in lattice dynamical systems. Nonlinearity 18 (2005) 631-657. 